Research Article

\title{
Synchronization in a Novel Local-World Dynamical Network Model
}

\author{
Jianeng Tang and Peizhong Liu \\ College of Engineering, Huaqiao University, No. 269 Chenghuabei Road, Quanzhou, Fujian 362021, China \\ Correspondence should be addressed to Peizhong Liu; liupeizhong_1234@126.com
}

Received 13 February 2014; Accepted 22 April 2014; Published 7 May 2014

Academic Editor: Wenwu Yu

Copyright (C) 2014 J. Tang and P. Liu. This is an open access article distributed under the Creative Commons Attribution License, which permits unrestricted use, distribution, and reproduction in any medium, provided the original work is properly cited.

\begin{abstract}
Advances in complex network research have recently stimulated increasing interests in understanding the relationship between the topology and dynamics of complex networks. In the paper, we study the synchronizability of a class of local-world dynamical networks. Then, we have proposed a local-world synchronization-optimal growth topology model. Compared with the local-world evolving network model, it exhibits a stronger synchronizability. We also investigate the robustness of the synchronizability with respect to random failures and the fragility of the synchronizability with specific removal of nodes.
\end{abstract}

\section{Introduction}

Complex networks are the sets of interconnected large-scale nodes, in which a node is a fundamental unit that can have different meanings in different situations [1-15]. Complex networks were conventionally researched by graph theory, for which a complex network was described by a random graph, where the radical theory was introduced by Erdös and Rényi [3]. At present, Watts and Strogatz (WS) [4] introduced the conception of small-world networks to describe a transition from a regular lattice to a random graph. Based on the research of Barabási and Albert [5], empirical results display that many large-scale complex networks are scalefree. Remarkably, a scale-free network is essentially inhomogeneous; in other words, the majority of nodes have extremely few connections, but a small number of specific nodes have many connections.

As shown in [5], the BA network model captures the basic mechanism which causes the power-law degree distribution, but the model also has several limitations: it only predicts a fixed exponent in a power-law degree distribution, while the measured real networks' exponents actually vary mostly from 1 to 3, which can have non-power-law features such as exponential cutoffs or saturation for small variables. In order to overcome these limitations and further understand various microscopic processes under the influence of the network topology and evolution, the researchers have done many valuable works about the aspect. The evolution factors may include a kind of sides such as roughly different types of preferential attachments, growths, local events, and competitions. To some extent, some researchers studied a nonlinear preferential attachment scheme with the degree probability; some researchers studied the accelerated growth in a directed network; the others also investigated the competition aspect and the distance preference. Some researchers also studied the propagation mechanism and the synchronization principle of complex network models. For details, please refer to the relevant literature [6-15].

Synchronization of complex networks has been a subject of intensive research with potential applications the Internet, the World Wide Web, food webs, electric power grids, and cellular and metabolic networks. On one hand, some researchers proposed some novel control laws to study the synchronization of complex networks. A novel impulsive control law was proposed for synchronization of stochastic discrete complex networks [16]. In [17], a simple but effective pinning algorithm for reaching synchronization on a general complex dynamical network was proposed. In recent years, researchers discussed the consensus in multiagent dynamical systems [18-20]. On the other hand, network topology structure provides a powerful metaphor for describing sophisticated collaborative dynamics of many practical systems in essence. So some researchers proposed some new network models to study the synchronization of complex networks. 
Local-world evolving network model was proposed in [21]. It captured an important feature in the evolution of many realworld complex networks: preferential attachment mechanism works only within local-world instead of whole networkwide. The degree distribution of this local-world evolving network model represented a transition between that of an exponential network and of a power-law scaling network, and the synchronization robustness and fragility of the localworld network model also displayed a transition between the exponential and the scale-free ones. According to the localworld evolving model, Sun et al. [22] studied the statistical properties of networks constructed and found that localworld size $M$ had great effect on network's connectivity: bigger $M$ made the networks more heterogeneous in connectivity. A comprehensive multilocal-world model was proposed in [23]. Gu and Sun [24] proposed a local-world node deleting evolving network model. In this paper, the objective is to find a new local area network model in which there is stronger synchronizability. Thence, a local-world synchronizationoptimal dynamical network model is proposed to describe the topology of evolving networks. Compared with the localworld evolving network model, the proposed network model exhibits a stronger synchronizability.

The rest of the paper is organized as follows: Section 2 introduces synchronization stability criterion of complex dynamical networks. Then, we study a brief summary about the local-world evolving network model and propose the novel local-world dynamical network model followed by some discussions on its synchronization robustness and fragility in Section 4. Finally, Section 5 concludes the investigation.

\section{Synchronization Stability Criterion of Complex Dynamical Networks}

Consider a dynamical network consisting of $N$ linearly and diffusively identical coupled nodes, with each node being an $n$-dimensional dynamical system. The state equations of the network can be written as $[6,25]$.

$$
\dot{x}_{i}=f\left(x_{i}\right)+c \sum_{j=1}^{N} a_{i j} \Gamma x_{j}, \quad i=1,2, \ldots, N
$$

where $x_{i}=\left(x_{i 1}, x_{i 2}, \ldots, x_{i n}\right) \in R^{n}$ are the state variables of node $i$ and the constant $c>0$ represents the coupling strength. For simplicity, we take $\Gamma=\operatorname{diag}\left\{r_{1}, r_{2}, \ldots, r_{n}\right\} \in$ $R^{n \times n}$ and $\Gamma$ is a diagonal matrix with $r_{i}=1$, for a particular $i$, and $r_{i}=0$, for $j \neq i$. If there is a connection between node $i$ and node $j(i \neq j)$, then $a_{i j}=a_{j i}=1$; otherwise, $a_{i j}=a_{j i}=$ $0(i \neq j)$. We take

$$
a_{i i}=-\sum_{j=1, j \neq i}^{N} a_{i j}=-k_{i}, \quad i=1,2, \ldots, N
$$

where the degree $k_{i}$ of node $i$ is defined to be the number of connection incidents on node $i$.
The coupling matrix $A=\left(a_{i j}\right)_{N \times N} \in R^{N \times N}$ represents the coupling configuration of the network. Suppose that the network is connected in the sense that there are no isolate clusters. The $A$ is a symmetric and irreducible matrix. In this case, it can be shown that zero is an eigenvalue of $A$ with multiplicity 1 and all the other eigenvalues of $A$ are strictly negative [26].

Dynamical network (1) is said to be (asymptotically) synchronized, if

$$
x_{1}(t)=x_{2}(t)=\cdots x_{N}(t)=s(t) \text { as } t \longrightarrow \infty,
$$

where $s(t)$ is a solution of an isolated node, which can be an equilibrium point, a periodic orbit, or a chaotic attractor, depending on the interest of study.

Let $0=\lambda_{1}>\lambda_{2} \geq \lambda_{3} \geq \cdots \lambda_{N}$ be the eigenvalues of the coupling matrix $A$. Consider network (1); suppose that there exist an $n \times n$ diagonal matrix $\Lambda>0$ and two constants $\bar{d}<0$ and $\tau>0$, such that

$$
[D f(s(t))+d \Gamma]^{T} \Lambda+\Lambda[D f(s(t))+d \Gamma] \leq-\tau I_{n},
$$

for all $d \leq \bar{d}$, where $I_{n} \in R^{n \times n}$ is an unit matrix and $D f(s(t))$ is the Jacobian of $f$ at $s(t)$. It has been shown that the synchronized state (3) is exponentially stable, if [12]

$$
c \geq\left|\frac{\bar{d}}{\lambda_{2}}\right| \text {. }
$$

Note that the criterion (5) may not hold, if the dynamical equations of a network cannot be written in the form of (2) [27].

Given the dynamics of an isolated node, the synchronizability of network (1) with respect to a specific coupling configuration $A$ is said to be strong, if the network can synchronize with a small coupling strength $c$. Inequality (5) implies that the synchronizability of network (1) can be characterized by the second-largest eigenvalue of its coupling matrix; that is, the smaller the second-largest eigenvalue, the stronger the synchronizability of a network.

Duan et al. analyzed complex network synchronizability using the theory of subgraphs and complementary graphs $[28,29]$. As shown in the literature [30], the local stability of the synchronized solution can be determined by analyzing the so-called master stability equation. If the synchronized region is an unbounded sector $(-\infty, \alpha)$, the eigenvalue $\lambda_{2}$ of $A$ determines the synchronizability [25]; if the synchronized region is a bounded sector $\left[\alpha_{1}, \alpha_{2}\right]$, the ratio $r(A)=\lambda_{2} / \lambda_{N}$ determines the synchronizability [27]. And Duan et al. proposed a design method for the inner linking matrix of rank 1 such that the resultant network had an unbounded synchronized region because an unbounded synchronized region was always easier to analyze than a bounded synchronized region. Obviously, the synchronized region is unbounded in our paper. In the paper [31], the authors proposed novel criteria of synchronization state for both delay-independent and delay-dependent stabilities of linear time-delay systems. 


\section{Local-World Dynamical Network Models}

3.1. The Local-World Evolving Network Model. In many reallife networks, owing to the existence of the local-world connectivity discussed above, each node in a network has only local connections and, therefore, only owns local information about the entire network. To model such a local-world effect, a local-world evolving network model (LW) is proposed and to be generated by the following algorithm [21].

(i) Start with a small number $m_{0}$ of nodes and small number $e_{0}$ of edges.

(ii) Select $M$ nodes randomly from the existing network, referred to as the "local world" of the new coming node.

(iii) Add a new node with $m$ edges, linked to $m$ nodes in its local world determined in (ii), using a preferential attachment with probability $\Pi\left(k_{i}\right)$ defined at every time step $t$ by

$$
\prod\left(k_{i}\right)=\frac{M}{m_{0}+t} \frac{k_{i}}{\sum_{j \in \text { local }} k_{j}} .
$$

After $t$ time steps, this procedure results in a network with $N=t+m_{0}$ nodes and $E=e_{0}+m t$ edges.

\subsection{The Local-World Node Deleting Evolving Network Model.} In the local-world node deleting evolving network (LWD network), an undirected and unweighted network is initialized with a small number $m_{0}$ of isolated nodes. The network is evolved with the following scheme [24].

At each time step $t$, either we act (i) with probability $p_{a}$ or we act (ii) with probability $1-p_{a}$.

(i) Node adding: the addition is achieved as follows:

(1) growth: add a new node with $m\left(m<m_{0}\right)$ edges connected to the network;

(2) local-world establishment: randomly select $M$ nodes from the whole network as the local world;

(3) preferential attachment: add $m$ edges between the new coming node and $m$ existing nodes in the local-world; the probability for node $i$ selected in the local world is

$$
\prod\left(k_{i}\right)=\frac{M}{N(t)} \frac{k_{i}}{\sum_{j \in \text { local }} k_{j}},
$$

where $N(t)$ is the total number of nodes after $t$ time steps.

(ii) Node deleting: delete a node from the network randomly and remove all the edges once attached to the deleting node.

3.3. The Local-World Synchronization-Optimal Dynamical Network Model. In this paper, a novel local-world dynamical network model called the local-world synchronizationoptimal dynamical network model (LWSO) is proposed. Then, the new network generation algorithm of the model is as follows. (i) Start with a small number $m_{0}$ of nodes and small number $e_{0}$ of edges.

(ii) Select $M$ nodes randomly from the existing network, referred to as the "local world" of the new coming node.

(iii) Add a new node with $m$ edges, linked to $m$ nodes in its local world determined in (ii). The criterion for choosing the $m$ vertices to which the new vertex connects is to optimize the synchronizability of the obtained network or, equivalently, to minimize the second-largest eigenvalue of the corresponding coupling matrix.

There are $q\left(q=C_{M}^{m}\right)$ types about choosing $m$ nodes from $M$ nodes. After $t$ time steps this procedure results in a network with $N=t+m_{0}$ nodes and $E=e_{0}+m t$ edges.

The network topology has significant effects on its traffic protocols, searching algorithms, and even virus propagation; therefore, modeling the network topology is extremely important. Currently, there are quite some models proposed and applied to describe the network topological features and properties, such as the BA [5], LW [21], and LWD [24] models. For simplicity, we take $N=1000, m=3, m_{0}=10, M=10$, and $p_{a}=0.7$. The details are as shown in Figure 1 .

Donetti et al. proposed an optimization algorithm to minimize the eigenvalue ratio [32]. According to the paper, we know that the synchronizability of a network becomes stronger and stronger when the eigenvalue ratio is smaller and smaller. In the paper, the rewirings are accepted in the existing networks, such as random graph, small-world, linear chain, and scale-free. In our paper, we have studied a novel growth topology model. In the model, the preferential attachment is as follows: the criterion for choosing the $m$ vertices to which the new vertex connects is to optimize the synchronizability of the obtained network or, equivalently, to minimize the second-largest eigenvalue of the corresponding coupling matrix. Obviously, the preferential attachment is similar to the optimization algorithm. The difference between the preferential attachment and the optimization algorithm is as follows: the former is applied to the growth of networks but the latter is applied to the existing large-scale networks. But their main drawback is that the calculation of eigenvalues is slow.

We have known that the connectivity of the BA scale-free network is heterogeneous. Most vertices have few connections and a small number of vertices have many connections. We have also found that local-world networks are topological quasimulticenter networks. There is a number of the "hubs" which are almost connected with all of vertices, but most of the vertices have very few connections. However, what about the topological structure of this local-world synchronizationoptimal network model? It has been pointed out that the eigenvalues spectrum of complex networks provides information about their structural properties and the quantity $R=\left(\lambda_{1}-\lambda_{2}\right) /\left(\lambda_{2}-\lambda_{N}\right)$ measures the distance of the first eigenvalue from the remaining part of the spectral density normalized by the extension of the remaining part [33].

As shown in Figure 2, the figure represents the values of $R$ of the BA networks, the LW networks, the LWD networks, 


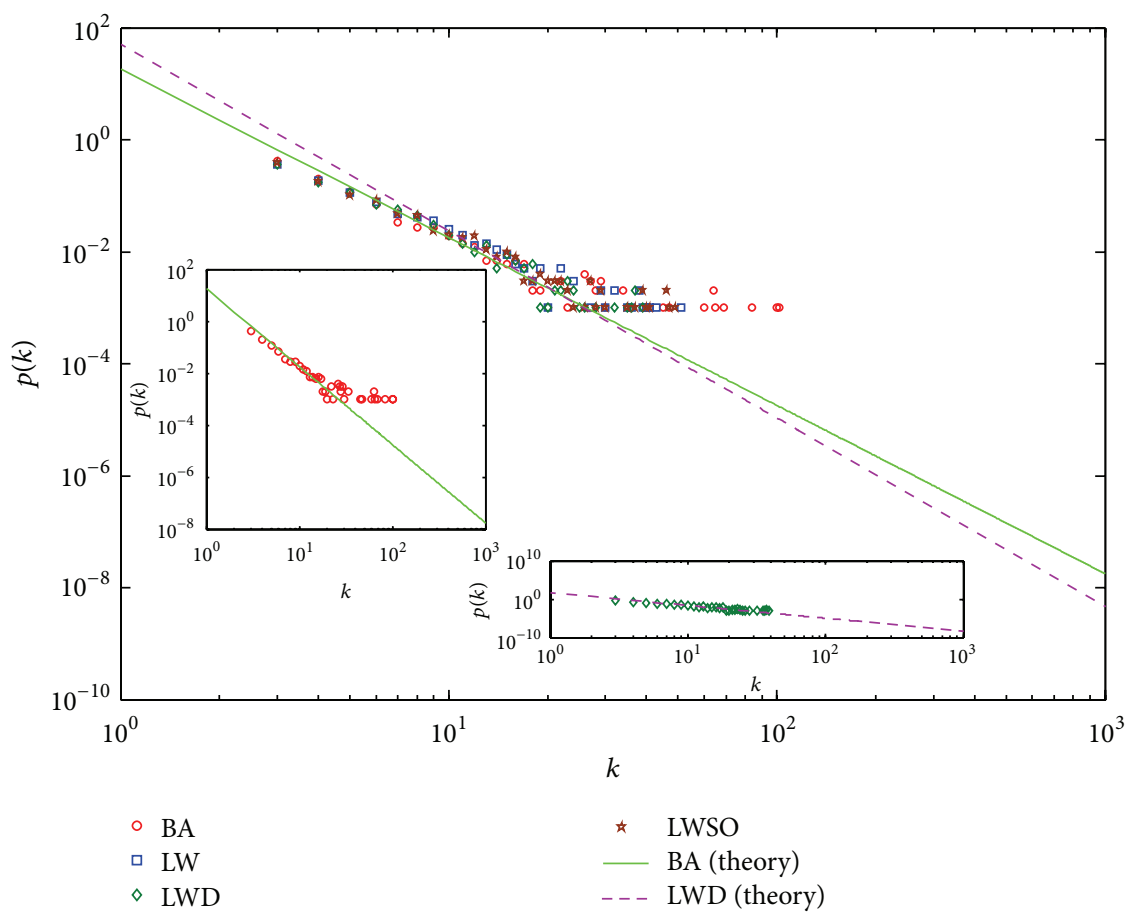

FIGURE 1: The degree distribution of nodes $p(k)$ of the BA networks (circles), the LW networks (squares), the LWD networks (diamonds), and the LWSO networks (pentagrams). In addition, the real line is $p(k)$ of BA model and the dash line is the theoretical prediction of $p(k)$ of LWD network. The right inset shows the degree distribution of the BA networks and the theoretical prediction; the left inset shows the LWD part.

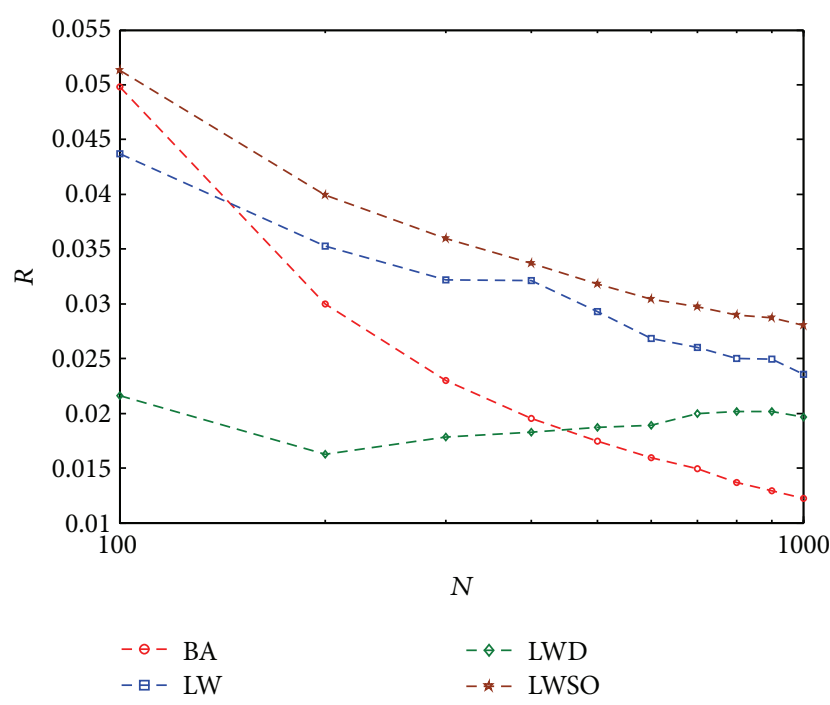

Figure 2: The values of $R$ for the BA networks (circles), the LW networks (squares), the LWD networks (diamonds), and the LWSO networks (pentagrams). Each curve in the figure is the average result of 5 groups of networks.

and the LWSO networks, while the size $N$ of networks ranges from 100 to 1000 . It can be observed that as the network size $N$ increases, values of $R$ of the three networks decay to converge to a power law. But values of $R$ of the LWD networks do not display the characteristic because the node deletion deletes a large portion of potential hubs during the network evolution. Then the value of $R$ of the LWSO dynamical network model changes more slowly. It explains that $R$ spans are the widest in the three models. The variation tendencies of $R$ correlate with different values of $m$, which increase as $m$ increases in extensive simulations.

3.4. Synchronization in Dynamical Networks. For clarity, we take $M=m_{0}$ in the construction of the four models. Then $A_{\mathrm{BA}}, A_{\mathrm{LW}}, A_{\mathrm{LWD}}$, and $A_{\text {LWSo }}$ represent the coupling matrices of the dynamical network (1) with the BA evolving network model, the LW evolving network model, the LWD evolving network model, and the LWSO dynamical network model, respectively, which has $N$ nodes and $N(N-M)+$ $e_{0}$ connections. Let $\lambda_{2 \mathrm{BA}}, \lambda_{2 \mathrm{LW}}, \lambda_{2 \mathrm{LWD}}$, and $\lambda_{2 \mathrm{LWSO}}$ be the second-largest eigenvalues of $A_{\mathrm{BA}}, A_{\mathrm{LW}}, A_{\mathrm{LWD}}$, and $A_{\mathrm{LWSO}}$, respectively. In numerical computations, the eigenvalues are obtained by averaging the results of 5 runs. For a fixed value of $m$ and $M$, the phenomenon found out that $\lambda_{2 \mathrm{BA}}$ decreases to a negative constant $\bar{\lambda}_{2 \mathrm{BA}}$, as $N$ increases. At the same time, $\lambda_{2 \mathrm{LW}}, \lambda_{2 \mathrm{LWD}}$, and $\lambda_{2 \mathrm{LWSO}}$ decrease to a negative constant $\bar{\lambda}_{2 \mathrm{LW}}, \bar{\lambda}_{2 \mathrm{LWD}}$, and $\bar{\lambda}_{2 \mathrm{LWSO}}$, respectively. For simplicity, we take $N=1000, m=3$, and $M=10$. The details are as shown in "Table 1" and in Figure 3.

As shown in Figure 3, the four second-largest eigenvalues converge to four negative constants, as $N$ increases. It has been observed that the second-largest eigenvalue of the 
TABLE 1: A comparison of the second-largest eigenvalues of different coupling topologies.

\begin{tabular}{ccccccc}
\hline$N$ & $m$ & $M$ & $\bar{\lambda}_{2 \mathrm{BA}}$ & $\bar{\lambda}_{\text {2LW }}$ & $\bar{\lambda}_{2 \mathrm{LWD}}$ & $\bar{\lambda}_{2 \mathrm{LWSO}}$ \\
\hline 1000 & 3 & 10 & -1.2586 & -1.2205 & -0.7762 & -1.4052 \\
\hline
\end{tabular}

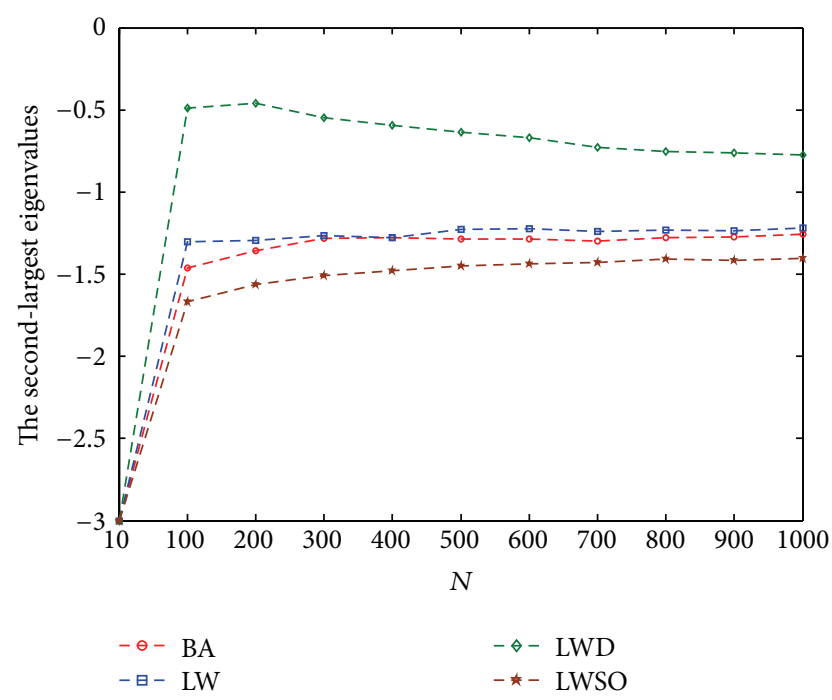

FIGURE 3: The second-largest eigenvalues of the coupling matrixes for the BA networks (circles), the LW networks (squares), the LWD networks (diamonds), and the LWSO networks (pentagrams). Each curve in the figure is the average result of 5 groups of networks.

LWSO network is smallest in the four networks, which indicates that the synchronizability of the LWSO network model is the strongest. With the exclusion of the LWD network, the eigenvalues of the other networks gradually become small. The eigenvalues of the LWD network are firstly larger and then they get smaller because the node deletion deletes a large portion of potential hubs during the network evolution.

Recently, more and more attention has been paid to the relation between the complex dynamic network topology characteristic and the network synchronizability by study scholars. The recent research work has discovered that many factors have had different influence on the network synchronizability, such as the maximum degree, the average way length, and the degree distribution. In [31], the effects of the maximum betweenness centrality $B_{\max }$ on the network synchronizability appear to be as follow: synchronizability is always improved, as $B_{\max }$ is reduced. Therefore, the betweenness centrality is proposed as a suitable indicator for predicting synchronizability on complex networks. On one hand, we can see that the second-largest eigenvalues of the LWSO network are smaller than those of the LW network as shown in Figure 3. Inequality (5) implies that the synchronizability of network (1) can be characterized by the second-largest eigenvalue of its coupling matrix; that is, the smaller the second-largest eigenvalue, the stronger the synchronizability of a network. Then, it is clear that the synchronizability of the LWSO network is stronger than that

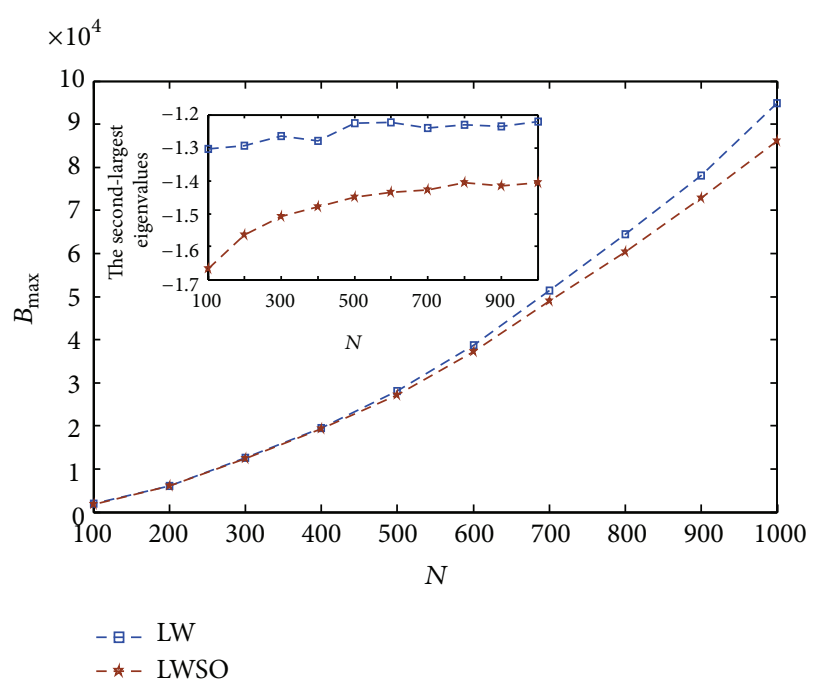

FIGURE 4: The values of $B_{\max }$ for the LW networks (squares) and the LWSO networks (pentagrams). The inset shows the corresponding second-largest eigenvalues. Each curve in the figure is the average result of 5 groups of networks.

of the LW network. On the other hand, we also notice, in Figure 4, that the maximum betweenness centrality of the LWSO network is smaller than that of the LW network. Accordingly, the view in [34] has been validated through the foregoing two simulations.

Here, it is worthwhile to emphasize that we have found some evidence indicating that there may exist some common features between synchronization and network traffic on a dynamical level [35-40]. Many previous works focus on the relationship between the distribution of the betweenness centrality and the capability of communication networks, with a latent assumption that the information packets go along the shortest paths from source to destination. Hence, the betweenness centrality is always considered as a static topological measure of networks. Here, we discover that this quantity is determined both by the routing algorithm and network topology; thus, one should pay more attention to the design of network topology. We believe this work may be helpful for understanding the intrinsic mechanism and the capability of network traffic.

\section{Robustness and Fragility}

Now, we consider the robustness of synchronization in dynamical network (1) against either random or specific removal of a small fraction $f(0<f<1)$ of the nodes in the network. Clearly, the removal of some nodes in a network (1) will change its coupling matrix. However, if the secondlargest eigenvalue of the coupling matrix remains unchanged, then the synchronizability of the network will also remain unchanged after the removal of some of its nodes.

Let $A \in R^{N \times N}$ and $B \in R^{(N-[f N]) \times(N-[f N])}$ be the coupling matrices of the original network with $N$ nodes and the new network after removal of $[f N]$ nodes, respectively. Denote $\lambda_{A 3}$ and $\lambda_{B 3}$ as the second-largest eigenvalues of $A$ and $B$, 
respectively. Suppose that nodes $i_{1}$ th, $i_{2}$ th, .., $i_{([f N])}$ th have been removed from the network. One can construct the new coupling matrix $B$ from the original coupling matrix $A$ as follows [6]:

(i) from the minor matrix $B \in R^{(N-[f N]) \times(N-[f N])}$ of $A$ by removing the $i_{1}$ th, $i_{2}$ th, .., $i_{[f N]}$ row-column pairs of A;

(ii) obtain $B=\left(b_{i j}\right)_{(N-[f N]) \times(N-[f N])}$ by recomputing the diagonal elements of the minor matrix $G=\left(g_{i j}\right)$ as follows:

$$
\begin{gathered}
g_{i j}=b_{i j}, \quad i \neq j ; \\
g_{i i}=-\sum_{j=1, j \neq i}^{N-[f N]} b_{i j}, \quad i=1,2, \ldots, N-[f N] .
\end{gathered}
$$

Here, we mainly compare the robustness and fragility between the LW network and the LWSO network. We know that the BA network is a global network. In addition, the node deletion will delete a large portion of potential hubs during the network evolution. It is harmful to the network evolution. In the further study, how to delete the nodes instead of random deletion is an important problem. In the simulation, for simplicity, we take $N=1000, m=3$, and $M=m_{0}=4,10$, respectively. Then we notice that "LW43" represents the LW model of $M=4$ and $m=3$. We also notice that "LWSO43" represents the LWSO dynamical network model of $M=4$ and $m=3$. At the same time, "LW103" and "LWSO103" represent the similar meaning. Then, those symbols stand for the same meaning in the following figures. The original network with the local-world or local-world synchronization-optimal topology contains 1000 nodes and about 3000 connections. We have known from [25] that the synchronizability of the original coupled network remains almost unchanged when a scale-free dynamical network has a random failure; for example, a very small fraction $f$ of nodes are randomly removed; however, the synchronizability of the original coupled network is also significantly decreased or even destroyed when the network is attacked intentionally; for example, a small fraction $f$ of "big" nodes are removed and then the original network changes significantly and even breaks into parts. These tell us that a scale-free network regarding its dynamical synchronization has the meanings of "robustness and yet fragility."

It has been shown, as in Figure 5, that the second-largest eigenvalues of the "LW103" have decreased from -1.2205 to -0.9046 when as many as $5 \%$ of the randomly chosen nodes are removed. Then, the decreased magnitude of the second-largest eigenvalues of the "LWSO103" is almost 32.83 percent. When more vertices are randomly removed, it is not significant that the reduction in the second-largest eigenvalue of the coupling matrix. This means that the "LW103" is more robust than the "LWSO103" against random failures. Then, we consider the fragility of the synchronizability with respect to deliberate attacks. A few isolated vertices or clusters may take place during the process of deliberate attacks. We remove the vertices with the highest degree and find that the secondlargest eigenvalues of the "LWSO103" reduce from -1.4052

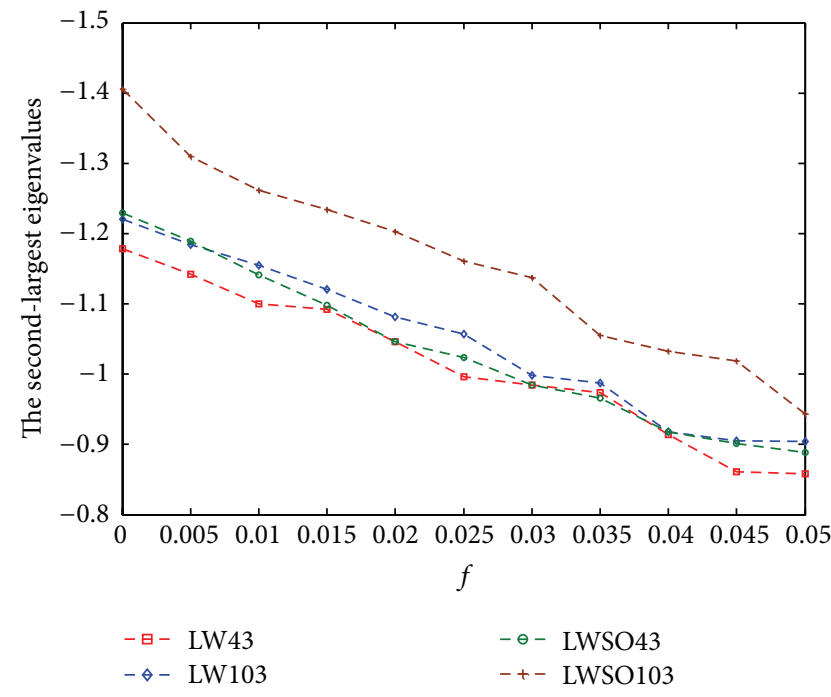

FIGURE 5: Synchronization robustness against random failures: changes of the second-largest eigenvalues of the local-world dynamical networks ( $M=4$, squares), the local-world synchronizationoptimal dynamical networks ( $M=4$, circles), the local-world dynamical networks ( $M=10$, diamonds), and the local-world synchronization-optimal dynamical networks ( $M=10$, pluses). Each curve in the figure is the average result of 5 groups of networks.

TABLE 2: A comparison of the decreased magnitudes of the secondlargest eigenvalues when $5 \%$ of the nodes are respect to random and specific removed.

\begin{tabular}{lcc}
\hline & Robustness & Fragility \\
\hline LW43 & $27.66 \%$ & $71.91 \%$ \\
LWSO43 & $27.73 \%$ & $67.01 \%$ \\
LW103 & $25.88 \%$ & $92.79 \%$ \\
LWSO103 & $32.83 \%$ & $86.5 \%$ \\
\hline
\end{tabular}

to -0.1895 when about $5 \%$ of the most connected vertices are removed. As shown in Figure 6, the decreased magnitude of the second-largest eigenvalues of "LW103" is about 92.79 percent. This implies that the "LWSO103" is less vulnerable to specific removal of those most connected vertices than the "LW103." At the same time, the comparison situation between the "LW43" and the "LWSO43" is similar, but the analysis result is less obvious than the above analysis result. The details are as shown in "Table 2." To sum up, the local-world network is particularly well suited to tolerate random errors compared with the local-world synchronization-optimal dynamical network. However, the latter is particularly well suited to tolerate intentional attacks compared with the former.

\section{Conclusion}

In the past decade, researchers have gained the significant progress about the effect of network topology on network synchronization dynamical behavior. In the paper, we study the synchronizability of a class of local-world dynamical networks. Then, we have proposed a local-world 


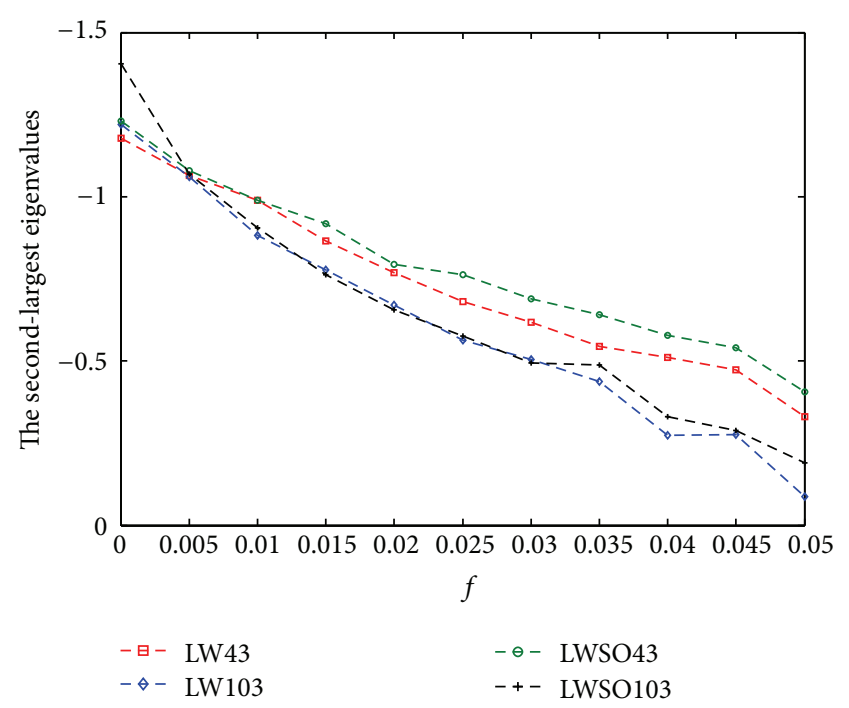

FIGURE 6: Synchronization fragility against specific attacks: changes of the second-largest eigenvalues of the local-world dynamical networks ( $M=4$, squares), the local-world synchronizationoptimal dynamical networks ( $M=4$, circles), the local-world dynamical networks ( $M=10$, diamonds), and the local-world synchronization-optimal dynamical networks ( $M=10$, pluses). Each curve in the figure is the average result of 5 groups of networks.

synchronization-optimal growth topology model. Compared with the local-world evolving network model, it exhibits stronger synchronizability. We have found some evidence indicating that there may be some common features between synchronization and network traffic on a dynamical level. So this work may be helpful for understanding the intrinsic mechanism and the capability of network traffic. Then, we also investigate the robustness of the synchronizability with respect to random failures and the fragility of the synchronizability with specific removal of nodes. We know that the localworld network is particularly well suited to tolerate random errors compared to the local-world synchronization-optimal dynamical network according to the simulation. However, the latter is particularly well suited to tolerate intentional attacks compared with the former.

\section{Conflict of Interests}

The authors declare that there is no conflict of interests regarding the publication of this paper.

\section{Acknowledgments}

This work was supported by the Natural Science Foundation of China under Grant no. 61231002 and no. 51075068, by Cloud Computing Platform for Internet of Things, Fujian Scientific Research Platform for Innovation under Grant no. 2013H2002, by the Fundamental Research Funds for the Central Universities under Grant no. JB-ZR1202, by the Foundation of Quanzhou under Grant no. 24201305, and by the Foundation of Huaqiao University under Grants no.
12BS228, no. 12Y0316, and no. 13BS103. The authors would like to thank the reviewers for their valuable suggestions and comments.

\section{References}

[1] S. H. Strogatz, "Exploring complex networks," Nature, vol. 410, pp. 268-276, 2001.

[2] R. Albert and A.-L. Barabási, "Statistical mechanics of complex networks," Reviews of Modern Physics, vol. 74, no. 1, pp. 47-97, 2002.

[3] P. Erdös and A. Rényi, "On the evolution of random graphs," Publications of the Mathematical Institute of the Hungarian Academy of Sciences, vol. 5, pp. 17-61, 1959.

[4] D. J. Watts and S. H. Strogatz, "Collective dynamics of "smallworld” networks," Nature, vol. 393, pp. 440-442, 1998.

[5] A.-L. Barabási and R. Albert, "Emergence of scaling in random networks," American Association for the Advancement of Science. Science, vol. 286, no. 5439, pp. 509-512, 1999.

[6] J. Fan and X. F. Wang, "On synchronization in scale-free dynamical networks," Physica A, vol. 349, no. 3-4, pp. 443-451, 2005.

[7] A. Arenas, D.-G. Albert, J. Kurths, Y. Moreno, and C. S. Zhou, "Synchronization in complex networks," Physics Reports, vol. 469, no. 3, pp. 93-153, 2008.

[8] J. N. Tang, C. R. Zou, L. Zhao, X. Z. Xu, and X. M. Du, "Impulsive stabilization for control and synchronization of complex networks with coupling delays," Journal of the Physical Society of Japan, vol. 81, Article ID 014003, 7 pages, 2012.

[9] M. J. Van den Hof Paul, D. Arne, S. C. H. Peter, and B. Xavier, "Identification of dynamic models in complex networks with prediction error methods-Basic methods for consistent module estimates," Automatica, vol. 49, no. 10, pp. 2994-3006, 2013.

[10] C.-H. Li, C.-C. Tsai, and S.-Y. Yang, "Analysis of epidemic spreading of an SIRS model in complex heterogeneous networks," Communications in Nonlinear Science and Numerical Simulation, vol. 19, no. 4, pp. 1042-1054, 2014.

[11] J. N. Tang, C. R. Zou, S. P. Wang, L. Zhao, and X. P. Liu, "Chaos synchronization of chen systems with time-varying delays," International Journal of Bifurcation and Chaos, vol. 22, Article ID 1250183, 8 pages, 2012.

[12] G. Bianconi and A. L. Barabási, "Competition and multiscaling in evolving networks," Europhysics Letters, vol. 54, no. 4, p. 436, 2001.

[13] L. K. Tang, J. Lu, and G. Chen, "Synchronizability of small-world networks generated from ring networks with equal-distance edge additions ," Chaos, vol. 22, Article ID 023121, 2012.

[14] G. H. Zhu, X. C. Fu, and G. Chen, "Spreading dynamics and global stability of a generalized epidemic model on complex heterogeneous networks," Applied Mathematical Modelling, vol. 36, no. 12, pp. 5808-5817, 2012.

[15] L. K. Tang, J. Lu, J. Lü, and X. Q. Wu, "Impact of node dynamics parameters on topology identification of complex dynamical networks," Nonlinear Dynamics, vol. 73, no. 1-2, pp. 1081-1097, 2013.

[16] C. J. Li, W. W. Yu, and T. W. Huang, "Impulsive synchronization schemes of stochastic complex networks with switching topology: average time approach " Neural Networks, vol. 54, pp. 8594, 2014.

[17] W. W. Yu, G. R. Chen, J. H. Lü, and J. Kurths, "Synchronization via pinning control on general complex networks," SIAM 
Journal on Control and Optimization, vol. 51, no. 2, pp. 13951416, 2013.

[18] Y. Zhao, Z. S. Duan, G. H. Wen, and Y. J. Zhang, "Distributed finite-time tracking control for multi-agent systems: an observer-based approach," Systems \& Control Letters, vol. 62, no. 1, pp. 22-28, 2013.

[19] W. W. Yu, G. R. Chen, and M. Cao, "Some necessary and sufficient conditions for second-order consensus in multi-agent dynamical systems," Automatica, vol. 46, no. 6, pp. 1089-1095, 2010.

[20] Y. Zhao, Z. Li, and Z. Duan, "Distributed consensus tracking of multi-agent systems with nonlinear dynamics under a reference leader," International Journal of Control, vol. 86, no. 10, pp. 18591869, 2013.

[21] X. Li and G. Chen, "A local-world evolving network model," Physica A, vol. 328, no. 1-2, pp. 274-286, 2003.

[22] S. W. Sun, Z. X. Liu, Z. Q. Chen, and Z. Z. Yuan, "Error and attack tolerance of evolving networks with local preferential attachment," Physica A, vol. 373, pp. 851-860, 2007.

[23] Z. P. Fan, G. Chen, and Y. N. Zhang, "A comprehensive multilocal-world model for complex networks," Physics Letters A, vol. 373, no. 18-19, pp. 1601-1605, 2009.

[24] Y. Y. Gu and J. T. Sun, "A local-world node deleting evolving network model," Physics Letters A, vol. 372, no. 25, pp. 45644568, 2008.

[25] X. F. Wang and G. R. Chen, "Synchronization in scale-free dynamical networks: robustness and fragility," IEEE Transactions on Circuits and Systems I: Fundamental Theory and Applications, vol. 49, no. 1, pp. 54-62, 2002.

[26] C. W. Wu and L. O. Chua, "Synchronization in an array of linearly coupled dynamical systems," IEEE Transactions on Circuits and Systems I Fundamental Theory and Applications, vol. 42, no. 8, pp. 430-447, 1995.

[27] M. Barahona and L. M. Pecora, "Synchronization in smallworld systems," Physical Review Letters, vol. 89, Article ID 054101, 2002.

[28] Z. S. Duan, G. R. Chen, and L. Huang, "Complex network synchronizability: analysis and control," Physical Review E, vol. 76, Article ID 056103, 2007.

[29] Z. S. Duan, C. Liu, and G. R. Chen, "Network synchronizability analysis: the theory of subgraphs and complementary graphs," Physica D, vol. 237, no. 7, pp. 1006-1012, 2008.

[30] L. M. Pecora and T. L. Carroll, "Master stability functions for synchronized coupled systems," Physical Review Letters, vol. 80, p. 2109, 1998.

[31] Q. Y. Wang, G. R. Chen, Q. S. Lu, and F. Hao, "Novel criteria of synchronization stability in complex networks with coupling delays," Physica A, vol. 378, no. 2, pp. 527-536, 2007.

[32] L. Donetti, P. I. Hurtado, and M. A. Muñoz, "Entangled networks, synchronization, and optimal network topology," Physical Review Letters, vol. 95, Article ID 188701, 2005.

[33] I. J. Farkas, I. Derenyi, A. L. Barabasi, and T. Vicsek, "Spectra of "real-world" graphs: beyond the semicircle law," Physical Review E, vol. 64, Article ID 026704, 2001.

[34] T. Nishikawa, A. E. Motter, Y.-C. Lai, and F. C. Hoppensteadt, "Heterogeneity in oscillator networks: are smaller worlds easier to synchronize?" Physical Review Letters, vol. 91, Article ID 014101, 2003.

[35] A. E. Motter, C. Zhou, and J. Kurths, "Network synchronization, diffusion, and the paradox of heterogeneity," Physical Review E, vol. 71, Article ID 016116, 2005.
[36] M. Chavez, D.-U. Hwang, A. Amann, H. G. E. Hentschel, and S. Boccaletti, "Synchronization is enhanced in weighted complex networks," Physical Review Letters, vol. 94, Article ID 218701, 2005.

[37] H.-T. Zhang, T. Yu, J.-P. Sang, and X.-W. Zou, "Dynamic fluctuation model of complex networks with weight scaling behavior and its application to airport networks "' Physica A, vol. 393, pp. 590-599, 2014.

[38] M. Zhao, T. Zhou, B.-H. Wang, and W.-X. Wang, "Enhanced synchronizability by structural perturbations," Physical Review E, vol. 72, Article ID 057102, 2005.

[39] T. Zhou, M. Zhao, and B.-H. Wang, "Better synchronizability predicted by crossed double cycle," Physical Review E, vol. 73, Article ID 037101, 2006.

[40] C.-Y. Yin, B.-H. Wang, W.-X. Wang, T. Zhou, and H.-J. Yang, "Efficient routing on scale-free networks based on local information," Physics Letters A, vol. 351, no. 4-5, pp. 220-224, 2006. 


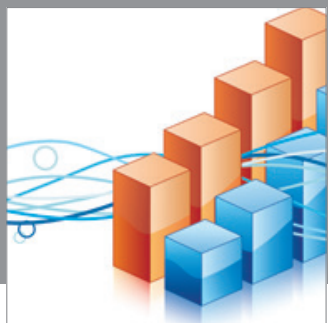

Advances in

Operations Research

mansans

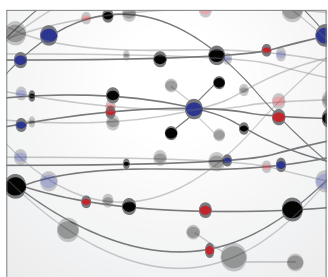

The Scientific World Journal
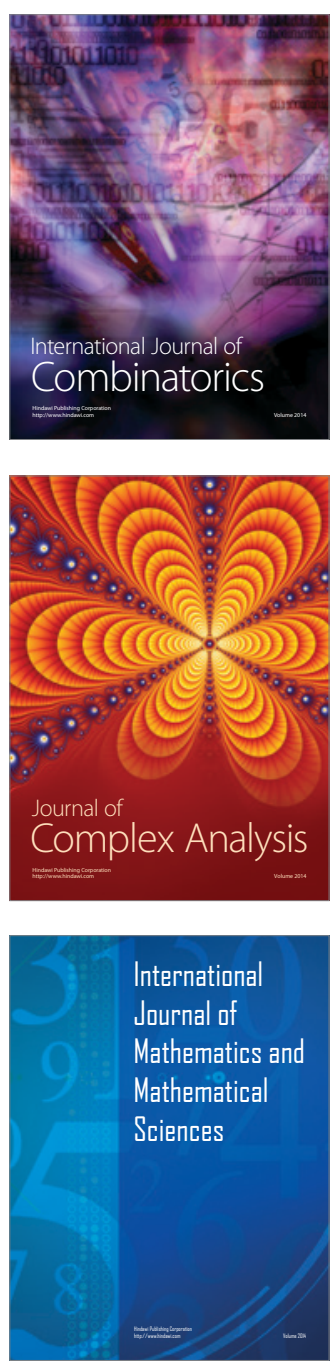
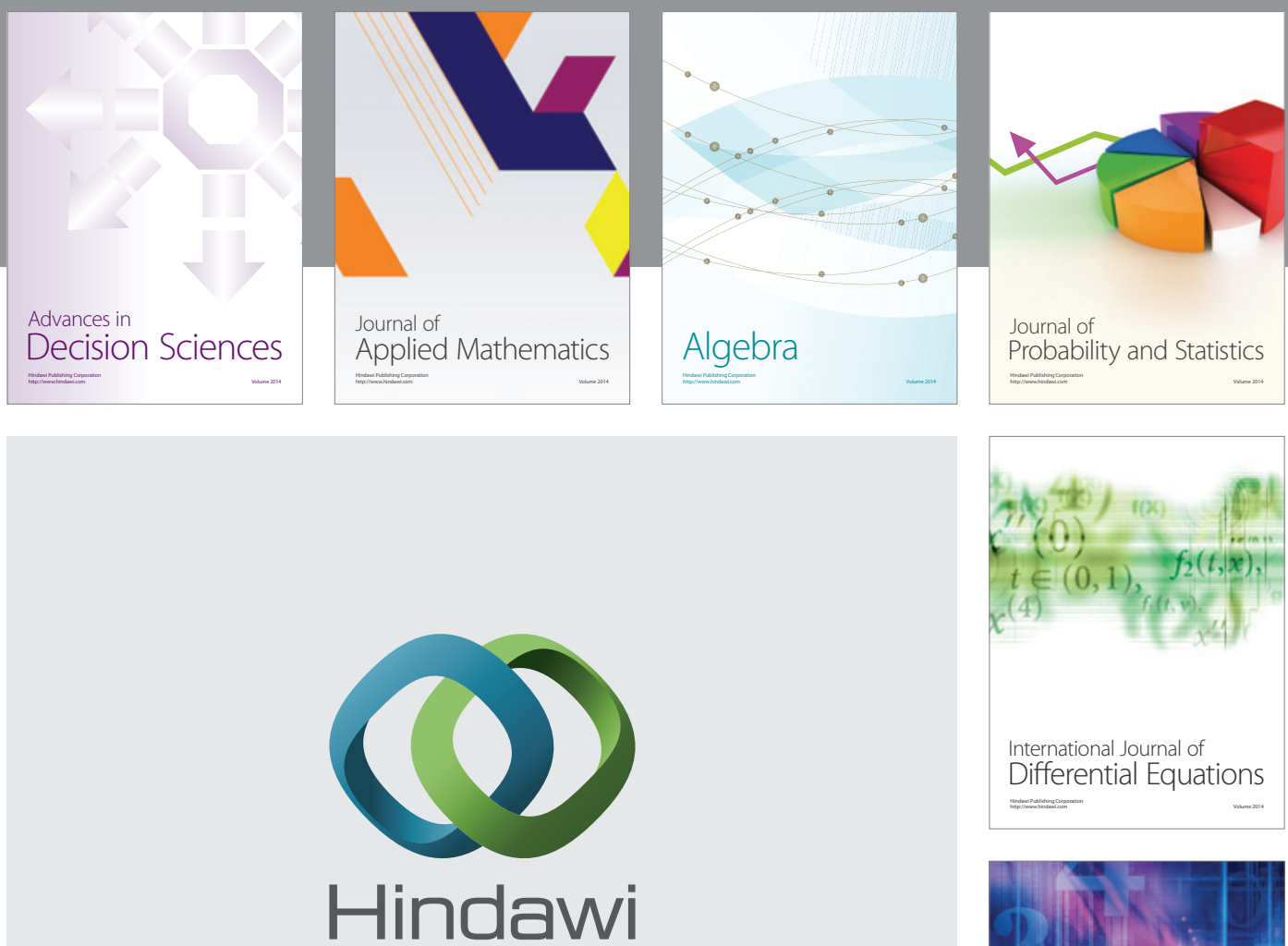

Submit your manuscripts at http://www.hindawi.com
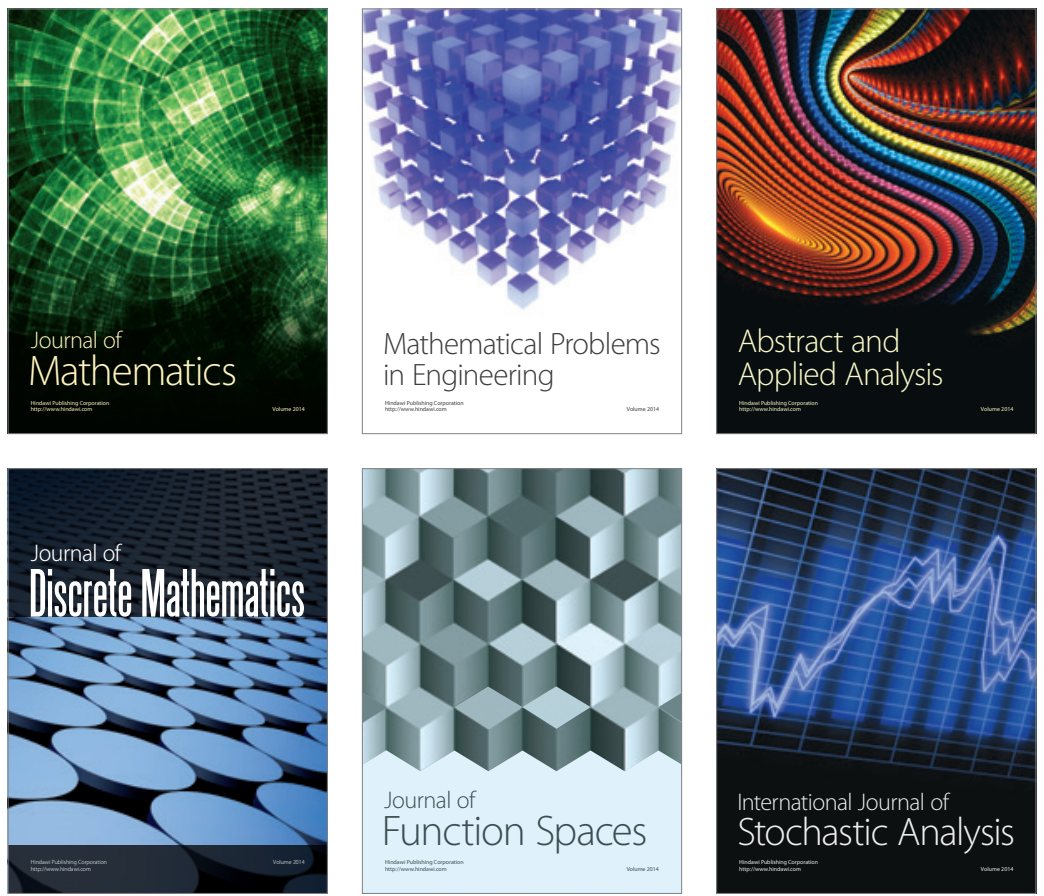

Journal of

Function Spaces

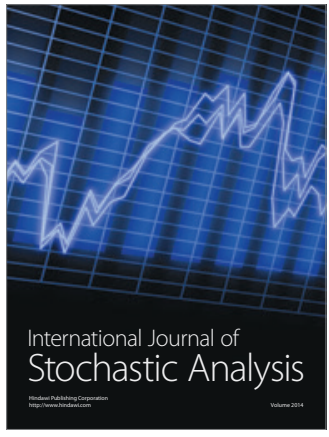

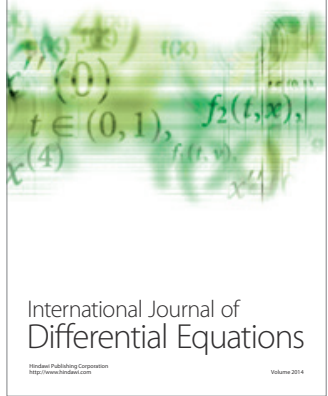
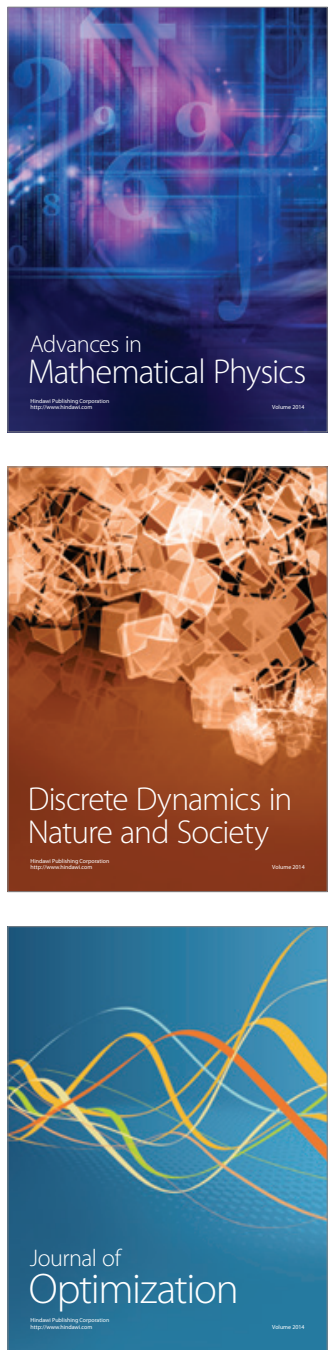